Bull. Austral. Math. Soc.

$11 \mathrm{~s} 40,11 \mathrm{~s} 25$

VOL. 56 (1997) [331-341]

\title{
FUNDAMENTAL THEOREM OF PREHOMOGENEOUS VECTOR SPACES OF CHARACTERISTIC $p$
}

\author{
Tatsuo Kimura, Takeyoshi Kogiso and Makiko Fujinaga
}

\begin{abstract}
For a local field of characteristic 0 , the functional equations of zeta distributions of prehomogeneous vector spaces have been obtained by M. Sato, Shintani, Igusa, F. Sato and Gyoja. In this paper, we shall consider the case of local fields of characteristic $\mathrm{p}>0$.
\end{abstract}

\section{1. $K$-REgUlar P.V.'S}

We fix a local field $K$ of characteristic $p>0$. Let $G$ be a connected linear algebraic group, $\rho$ its rational representation of $G$ on a finite-dimensional vector space $V$, all defined over an algebraic closure $\bar{K}$ of $K$. We call a triplet $(G, \rho, V)$ a prehomogeneous vector space (abbreviated P.V.) if $V$ has a Zariski-dense $G$-orbit $Y$.

Any point of $Y$ is called a generic point and the isotropy subgroup $G_{y}=\{g \in$ $G ; \rho(g) y=y\}$ of a generic point $y$ is called a generic isotropy subgroup. Note that we have $\operatorname{dim} G_{y}=\operatorname{dim} G-\operatorname{dim} V$ if and only if $y \in Y$. A non-zero rational function $f(x)$ on $V$ is called a relative invariant of $(G, \rho, V)$ if $f(\rho(g) x)=\chi(g) f(x)$ holds for any $g \in G$ and $x \in Y$ where $\chi: G \rightarrow G L_{1}$ is a rational character of $G$.

The complement $S$ of $Y$ is a Zariski-closed set which is called the singular set of the P.V. $(G, \rho, V)$. Now we assume that $(G, \rho, V)$ is defined over $K$, that is, $G, \rho, V$ are all defined over $K$. Let $S_{i}=\left\{x \in V ; f_{i}(x)=0\right\}(i=1, \ldots, l)$ be the $K$-irreducible component of the $K$-rational points $S_{K}$ of $S$ of codimension one defined by a $K$-irreducible (not necessarily absolutely irreducible) polynomial $f_{i}(x)(i=1, \ldots, l)$. Then $f_{1}(x), \ldots, f_{l}(x)$ are algebraically independent relative invariants and any relative invariant $f(x)$ in $K(V)$ is of the form $f(x)=$ $c \cdot f_{1}(x)^{m_{1}} \ldots f_{l}(x)^{m_{l}}\left(c \in K^{\times},\left(m_{1}, \ldots, m_{l}\right) \in \mathbf{Z}^{l}\right)$. We call $f_{1}(x), \ldots, f_{l}(x)$ the basic $K$-relative invariants of $(G, \rho, V)$. Let $\chi_{i}$ be the rational character of $G$ corresponding to $f_{i}(i=1, \ldots, l)$. Let $X(G)_{K}$ be the group of $K$-rational characters of $G, X_{1}(G)_{K}$ its subgroup corresponding to $K$-relative invariants. Then $X_{1}(G)_{K}$ is a free Abelian group of rank $l$ generated by $\chi_{1}, \ldots, \chi_{l}$.

Received 9th December, 1996

We would like to express our hearty thanks to Professor M. Kashiwara and Professor A. Gyoja who gave us invaluable suggestions for our research.

Copyright Clearance Centre, Inc. Serial-fee code: 0004-9729/97 $\$$ A2.00+0.00. 
Let $G_{1}$ be a subgroup of $G$ generated by the commutator subgroup $[G, G]$ and a generic isotropy subgroup. This does not depend on the choice of a generic point. For $\chi \in X(G)_{K}$, it is in $X_{1}(G)_{K}$ if and only if $\left.\chi\right|_{G_{1}}=1$. For a relative invariant $f(x)$ of $(G, \rho, V)$, we can define a rational map $\varphi_{f}: Y \rightarrow V^{*}$ by

$$
\varphi_{f}(x)={ }^{t}\left(\frac{1}{f(x)} \cdot \frac{\partial f}{\partial x_{1}}(x), \ldots, \frac{1}{f(x)} \cdot \frac{\partial f}{\partial x_{n}}(x)\right)
$$

where $V^{*}$ is the dual vector space of $V$ and we identify $V$ and $V^{*}$ with $\bar{K}^{n}$ by taking a pair of dual bases. This definition does not depend on the choice of bases. We sometimes denote $\varphi_{f}(x)$ by $\operatorname{grad} \log f(x)$. By a direct calculation, we have

(1) $\varphi_{f}(\rho(g) x)=\rho^{*}(g) \varphi_{f}(x)$ for $g \in G$ and $x \in Y$ where $\rho^{*}$ denotes the contragredient representation of $\rho$, and

(2) $\left\langle d \rho(A) x, \varphi_{f}(x)\right\rangle=d \chi(A)$ for $x \in Y$ and $A \in \operatorname{Lie}(G)$ where $d \rho$ (respectively $d \chi$ ) is the infinitesimal representation of $\rho$ (respectively the infinitesimal character of $\chi$ ) of the Lie algebra Lie $(G)$ of $G$.

Definition 1.1: A relative invariant is called non-degenerate if $\varphi_{f}: Y \longrightarrow V^{*}$ is dominant. If there exists a non-degenerate relative invariant $f(x)$ in $K(V)$, we say that $(G, \rho, V)$ is a $K$-regular P.V. In this case, $\varphi_{f}(Y)$ is a Zariski-dense $G$-orbit in $V^{*}$, and hence the dual triplet $\left(G, \rho^{*}, V^{*}\right)$ is also a P.V. For a generic point $y \in Y^{*}$, the dominant morphism $\psi: G \longrightarrow V^{*}$ defined by $\psi(g)=\rho^{*}(g) y$ is called an open orbit morphism. If there is a non-degenerate relative invariant $f$ such that $\varphi_{f}: Y \rightarrow V^{*}$ is separable and also an open orbit morphism $\psi: G \longrightarrow V^{*}$ is separable, we say that $(G, \rho, V)$ is a separable $K$-regular P.V. In this case, the Hessian

$$
F(x)=\operatorname{det}\left(\frac{\partial^{2} \log f}{\partial x_{i} \partial x_{j}}(x)\right)
$$

of $\log f$ is a non-zero relative invariant corresponding to the character $\chi_{0}^{-1}$ with $\chi_{0}(g)=$ $\operatorname{det} \rho(g)^{2}$ for $g \in G$. Hence we have $\operatorname{det} \rho(g)^{2} \in X_{1}(G)_{K}$ for a separable $K$-regular P.V. In general,we denote by $Y_{K}, S_{K}$, et cetera, $K$-rational points of $Y, S$, et cetera. We write $X_{1}^{*}(G)_{K}$ (respectively $X^{*}(G)_{K}, Y^{*}, S^{*}$, et cetera) for $\left(G, \rho^{*}, V^{*}\right)$ which corresponds to $X_{1}(G)_{K}$ (respectively $X(G)_{K}, Y, S$, et cetera) for $(G, \rho, V)$.

Proposition 1.2. Assume that $(G, \rho, V)$ and $\left(G, \rho^{*}, V^{*}\right)$ are $K$-regular $P . V$.'s. Then we have the following assertion.

(1) $X_{1}(G)_{K}=X_{1}^{*}(G)_{K}$.

(2) For a non-degenerate $K$-relative invariant $f$, the $\operatorname{map} \varphi=\operatorname{grad} \log f$ : $Y \rightarrow Y^{*}$ is bijective. 
PROOF: Since $\varphi(Y)$ is a Zariski-dense $G$-orbit in $V^{*}$, we have $\varphi(Y)=Y^{*}$, that is, $\varphi$ is surjective. Since $\rho^{*}(g) \varphi(x)=\varphi(\rho(g) x)$, we have $G_{x} \subset G_{\varphi(x)}$ for $x \in Y$. Now let $f^{*}$ be a non-degenerate relative invariant in $K\left(V^{*}\right)$, and put $\varphi^{*}=\operatorname{grad} \log f^{*}$ : $Y^{*} \rightarrow Y$. Similarly we have $G_{y} \subset G_{x^{\prime}}$ for $y=\varphi(x)$ and $x^{\prime}=\varphi^{*}(y)$, and hence $G_{x} \subset G_{y} \subset G_{x^{\prime}}$. Since $x^{\prime}=\rho\left(g_{0}\right) x$ for some $g_{0} \in G$, we have $G_{x^{\prime}}=g_{0} G_{x} g_{0}^{-1} \supset G_{x}$. Since $\operatorname{dim} G_{x^{\prime}}=\operatorname{dim} G_{x}$, the algebraic group $G_{x^{\prime}}$ and $G_{x}$ have the same connected component $H$ of the identity. Since $G_{x^{\prime}}=g_{0} G_{x} g_{0}^{-1}$ is isomorphic to $G_{x}$, the numbers of their connected components coincide, that is, $\left[G_{x^{\prime}}: H\right]=\left[G_{x}: H\right]$ with $G_{x^{\prime}} \supset G_{x}$. This implies $G_{x^{\prime}}=G_{x}$, and hence $G_{x}=G_{y}$ with $y=\varphi(x)$.

Thus we have $G_{1}=G_{1}^{*}$ and hence $X_{1}(G)_{K}=X_{1}^{*}(G)_{K}$. Note that $X_{1}(G)_{K}=$ $\left\{\chi \in X(G)_{K} ;\left.\chi\right|_{G_{1}}=1\right\}$. Now assume that $\varphi\left(x_{1}\right)=\varphi\left(x_{2}\right)$ with $x_{2}=\rho(g) x_{1}$ for some $g \in G$. Then we have $\varphi\left(x_{1}\right)=\varphi\left(x_{2}\right)=\varphi\left(\rho(g) x_{1}\right)=\rho^{*}(g) \varphi\left(x_{1}\right)$ and hence $g \in G_{\varphi\left(x_{1}\right)}=G_{x_{1}}$, that is, $x_{2}=\rho(g) x_{1}=x_{1}$. Thus $\varphi$ is injective.

Proposition 1.3. Assume that $(G, \rho, V)$ and $\left(G, \rho^{*}, V^{*}\right)$ are $K$-regular $P$.V.'s. Then we have \# $\rho(G)_{K} \backslash Y_{K}=\# \rho^{*}(G)_{K} \backslash Y_{K}^{*}$.

Proof: Let $f$ be a non-degenerate relative invariant in $K(V)$ and put $\varphi=$ $\operatorname{grad} \log f$. Then for any $x \in Y_{K}$, we have $\varphi\left(\rho(G)_{K} \cdot x\right)=\rho^{*}(G)_{K} \cdot \varphi(x) \subset Y_{K}^{*}$, that is, $\varphi$ maps an orbit in $Y_{K}$ to an orbit in $Y_{K}^{*}$.

By Proposition 1.2, this map $\varphi$ is injective, and hence \# $\#(G)_{K} \backslash Y_{K} \leqq \# \rho^{*}(G)_{K} \backslash Y_{K}^{*}$. Similarly we have \# $\rho^{*}(G)_{K} \backslash Y_{K}^{*} \leqq \# \rho(G)_{K} \backslash Y_{K}$.

Proposition 1.4. Assume that $(G, \rho, V)$ is a separable $K$-regular $P . V$. Then its dual $\left(G, \rho^{*}, V^{*}\right)$ is also a separable $K$-regular P.V.

Proof: Let $f$ be a separable non-degenerate relative invariant in $K(V)$ and put $\varphi=\operatorname{grad} \log f: Y \rightarrow Y^{*}$. First we show that $\varphi$ is injective. Assume that $\varphi(x)=\varphi\left(x^{\prime}\right)$. Since $d \chi(A)=\langle d \rho(A) x, \varphi(x)\rangle=-\left\langle x, d \rho^{*}(A) \varphi(x)\right\rangle$, we have $\left\langle x-x^{\prime}, d \rho^{*}(A) \varphi(x)\right\rangle=0$ for all $A \in \operatorname{Lie}(G)$. Since $\psi: G \rightarrow V^{*}$ with $\psi(g)=\rho^{*}(g) \varphi(x)$ is separable, we have $\left\{d \rho^{*}(A) \varphi(x) ; A \in \operatorname{Lie}(G)\right\}=V^{*}$, and hence $x-x^{\prime}=0$, that is, $x=x^{\prime}$. For any $g \in G_{\varphi(x)}\left(\supset G_{x}\right)$, we have $\varphi(\rho(g) x)=\rho^{*}(g) \varphi(x)=\varphi(x)$. As $\varphi$ is injective, we have $\rho(g) x=x$, that is, $g \in G_{x}$. This implies that $G_{x}=G_{\varphi(x)}$ and hence $X_{1}(G)_{K}=X_{1}^{*}(G)_{K}$. A rational character $\chi$ corresponding to $f$ is in $X_{1}(G)_{K}$ and hence $\chi^{-1} \in X_{1}^{*}(G)_{K}$. This implies that there exists a relative invariant $f^{*}$ in $K\left(V^{*}\right)$ satisfying $f^{*}\left(\rho^{*}(g) y\right)=\chi(g)^{-1} f^{*}(y)$ for $g \in G$ and $y \in Y^{*}$.

Put $\varphi^{*}=\operatorname{grad} \log f^{*}$. Then we have $\left\langle\varphi^{*}(y), d \rho^{*}(A) y\right\rangle=-d \chi(A)$. Since $d \chi(A)=$ $\langle d \rho(A) x, \varphi(x)\rangle=-\left\langle x, d \rho^{*}(A) \varphi(x)\right\rangle$, we have $\left\langle x-\varphi^{*}(y), d \rho^{*}(A) y\right\rangle=0$ for $y=\varphi(x)$ and all $A \in \operatorname{Lie}(G)$.

Since the open orbit morphism $\psi$ is separable, we have $\left\{d \rho^{*}(A) y ; A \in \operatorname{Lie}(G)\right\}=$ $V^{*}$, and hence $\varphi^{*}(y)=x \in Y$, that is, $\varphi^{*}\left(Y^{*}\right)=Y$. This implies that $Y$ and $Y^{*}$ 
are biregularly isomorophic. Hence $f^{*}$ is separable non-degenerate, and an open orbit morphism $\psi^{*}: G \longrightarrow V$ is separable, that is, $\left(G, \rho^{*}, V^{*}\right)$ is a separable $K$-regular P.V.

Note that in the case of $\operatorname{ch}(K)=0$, a $K$-regular P.V. is automatically a separable $K$-regular P.V. and the proof of Proposition 1.4 gives the equivalence between $K$ regularity of $(G, \rho, V)$ and that of $\left(G, \rho^{*}, V^{*}\right)$.

Now we shall consider a sufficient condition to ensure that $\# \rho(G)_{K} \backslash Y_{K}$ is finite.

LEMMA 1.5. Let $X$ be a homogeneous space of the connected algebraic group $G$ over $\bar{K}$ where the action is defined over $K$. Let $K_{\text {sep }}$ be the separable closure of $K$ in $\bar{K}$. Then $X\left(K_{\text {sep }}\right)$ is a homogeneous space of $G\left(K_{\text {sep }}\right)$.

Proof: Since $X\left(K_{\mathrm{sep}}\right)$ is dense in $X=X(\bar{K})$ (see Borel [2]), we may take $x_{0} \in X\left(K_{\text {sep }}\right)$. Since the map $f: G \rightarrow X$ with $f(g)=g \cdot x_{0}$ is defined over $K_{\text {sep }}$, the fibre $f^{-1}(x)$ of each $x \in X\left(K_{\text {sep }}\right)$ is also defined over $K_{\text {sep }}$. Since the set $X(\bar{K})$ is homogeneous by $G(\bar{K})$, we have $f^{-1}(x)(\bar{K}) \neq \emptyset . f^{-1}(x)\left(K_{\text {sep }}\right)$ is dense in $f^{-1}(x)(\bar{K})$ and $f^{-1}(x)\left(K_{\text {sep }}\right) \subset G\left(K_{\text {sep }}\right)$ which implies $g \cdot x_{0}=x$. Thus $X\left(K_{\text {sep }}\right)$ is a homogeneous space of $G\left(K_{\mathrm{sep}}\right)$.

By this lemma, we can use Galois cohomology to investigate $X(K)$ as a $G(K)$ space.

Professor J.P. Serre kindly told us of the following theorem with the proof which was explained by Tits to him.

THEOREM 1.6. Let $K$ be a local field of characteristic $p>0$ (or more generally let $K$ be a field complete with respect to a discrete valuation, and with the residue field $k$ of type $(F)$ in the sense of Serre [18]). Let $G$ be a connected smooth reductive group over $K$. Then $H^{1}(K, G)$ is finite.

Proof: (After Serre's letter on 9th September 1992.) Let $K^{\prime}$ be the maximal unramified extension of $K$. The field $K^{\prime}$ is known to be of dimension at most 1. (in the sense of [18, II, Section 3]). By a theorem of Steinberg (for $K^{\prime}$ perfect) and of BorelSpringer (for $K^{\prime}$ imperfect - see [1, II, p.761] we have $H^{1}\left(K^{\prime}, G\right)=0$. Hence the Galois cohomology of $G$ over $K$ is killed by $K^{\prime}$, that is, it is equal to $H^{1}\left(K^{\prime} / K, G\right)$. We may now apply a theorem of Bruhat-Tits [3, Theorem 3.12]; this says that $H^{1}\left(K^{\prime} / K, G\right)$ is contained in a finite union of cohomology sets $H^{1}\left(k, G_{i}\right)$, where the $G_{i}$ 's are algebraic linear groups (not neccessarily connected) over $k$. Since $k$ is type $(\mathrm{F})$, each $H^{1}\left(k, G_{i}\right)$ is finite (see [1, II, p.404, Theorem 6.2], or [18, III, Theorem 4]. Hence $H^{1}(K, G)$ is finite.

Let B be a subgroup of $G L_{n}\left(K_{\text {sep }}\right)$ and A a normal subgroup of B. Then $G=$ $\operatorname{Gal}\left(K_{\mathrm{sep}} / K\right)$ acts on them. For a 1-cocycle $b \in Z^{1}(G, B)$, let ${ }_{b} A$ be the twist of A by $b=\left\{b_{s}\right\}$, that is, ${ }_{b} A \cong A$, but $\mathrm{G}$ acts on ${ }_{b} A$ by $s \circ a=b_{s} \cdot s(a) \cdot b_{s}^{-1}$ for $s \in G, a \in A$. 
We learned the following lemma from Professor A. Gyoja.

Lemma 1.7. There exists a bijection between $H^{1}(G, A)$ and $H^{1}\left(G,{ }_{b} A\right)$.

ProOF: Since $H^{1}\left(G, G L_{n}\right)=\{1\}$, there exists $a \in G L_{n}$ satisfying $b_{s}=a^{-1} \cdot s(a)$ $(s \in G)$. Define $f: G L_{n} \rightarrow G L_{n}$ by $f(x)=a^{-1} x a$. Then we have

$$
s \circ f(x)=b_{s} \cdot s\left(a^{-1} x a\right) \cdot b_{s}^{-1}=a^{-1} \cdot s(x) \cdot a=f(s(x)),
$$

and hence we can identify ${ }_{b} A=f(A)$. For $\left\{a_{s}\right\} \in Z^{1}(G, A)$, we make correspond $\left\{f\left(a_{s}\right)\right\} \in Z^{1}\left(G,{ }_{b} A\right)$. This gives a bijection between $H^{1}(G, A)$ and $H^{1}\left(G,_{b} A\right)$.

THEOREM 1.8. Let $G$ be a smooth reductive group over $K$, not necessarily connected. Then $H^{1}(K, G)$ is finite.

Proof: Let $G^{0}$ be a connected component of G. Then $G / G^{0}$ is a finite group. Since $1 \rightarrow G^{0} \rightarrow G \rightarrow G / G^{0} \rightarrow 1$ is exact, we have $H^{1}\left(K, G^{0}\right) \rightarrow H^{1}(K, G) \rightarrow$ $H^{1}\left(K, G / G^{0}\right)$ is exact as distinguished point sets. By [18, Chapter III, Section 4, Proposition 8], we have $\# H^{1}\left(K, G / G^{0}\right)<+\infty$. By Theorem 1.6, we have $\# H^{1}\left(K, G^{0}\right)<+\infty$. By Lemma 1.7 and [18, Proposition 35], we have $\# H^{1}(K, G)<$ $+\infty$.

Proposition 1.9. Let $(G, \rho, V)$ be a P.V. defined over $K$ with a reductive generic isotropy subgroup. Then $\# \rho(G)_{K} \backslash Y_{K}$ is finite.

Proof: Let $H$ be a generic isotropy subgroup of a point in $Y_{K}$. Then there exists a bijection between $\rho(G)_{K} \backslash Y_{K}$ and $\operatorname{Ker}\left(H^{1}(K, H) \rightarrow H^{1}(K, G)\right)$ (see Serre [18]). By Theorem 1.8, $H^{1}(K, H)$ is finite, and hence $\rho(G)_{K} \backslash Y_{K}$ is a finite set.

EXAMPLE 1.10. Let $G$ be the subgroup of $G L_{n}$ consisting of all lower triangular matrices. Let $V$ be the totality of symmetric $n \times n$ matrices and define $\rho$ by $\rho(g) x=$ $g x^{t} g$ for all $g \in G$ and $x \in V$. Since $\operatorname{dim} G=\operatorname{dim} V$, a generic isotropy subgroup is a finite subgroup and hence we have $\# \rho(G)_{K} \backslash Y_{K}=\nu<+\infty$ by Proposition 1.9.

Moreover $\operatorname{det} x$ is a non-degenerate $K$-relative invariant. By $\operatorname{tr}(x y)(x, y \in V)$, we identify $V$ with its dual $V^{*}$. Then $(G, \rho, V)$ and $\left(G, \rho^{*}, V^{*}\right)$ are $K$-regular P.V.'s. Hence, by Proposition 1.3, we have \# $\rho^{*}(G)_{K} \backslash Y_{K}^{*}=\nu<+\infty$.

Proposition 1.11. Let $(G, \rho, V)$ be an irreducible regular $P$.V. defined over $K$. Then we have $\# \rho(G)_{K} \backslash Y_{K}<+\infty$.

Proof: By a classification of irreducible P.V.'s (see Chen [5]), we know that a generic isotropy subgroup is reductive.

\section{ZETA DISTRIBUTIONS}

Let $K$ be a local field of characteristic $p>0$. Assume that $(G, \rho, V)$ and its dual $\left(G, \rho^{*}, V^{*}\right)$ are $K$-regular P.V.'s. Moreover we shall assume that $Y_{K}=Y_{1} \cup \cdots \cup Y_{\nu}$ 
decomposes into a finite union of $\rho(G)_{K^{-o r b i t s}} Y_{i}(1 \leqq i \leqq \nu)$, that is, \# $\rho(G)_{K} \backslash Y_{K}=$ $\nu<+\infty$. Then by Proposition 1.3, we have $Y_{K}^{*}=Y_{1}^{*} \cup \cdots \cup Y_{\nu}^{*}$.

Let $f_{1}(x), \ldots, f_{l}(x)$ (respectively $\left.f_{1}^{*}(y), \ldots, f_{l}^{*}(y)\right)$ be basic $K$-relative invariants of $(G, \rho, V)$ (respectively $\left(G, \rho^{*}, V^{*}\right)$ ). Let $\chi_{i}$ (respectively $\chi_{i}^{*}$ ) be the corresponding character of $f_{i}$ (respectively $f_{i}^{*}$ ). Then we have $X_{1}(G)_{K}=\left\langle\chi_{1}, \ldots, \chi_{l}\right\rangle$ and $X_{1}^{*}(G)_{K}=$ $\left\langle\chi_{1}^{*}, \ldots, \chi_{l}^{*}\right\rangle$.

By Proposition 1.2, we have $X_{1}(G)_{K}=X_{1}^{*}(G)_{K}$ so that there exists a unique matrix $U=\left(u_{i j}\right) \in G L_{l}(\mathbf{Z})$ satisfying $\chi_{i}=\prod_{j=1}^{l} \chi_{j}^{* u_{i j}}$. Since $\operatorname{det} \rho(g)^{2} \in X_{1}(G)_{K}$, we have $\operatorname{det} \rho(g)^{2}=\chi_{1}^{2 \lambda_{1}} \ldots \chi_{l}^{2 \lambda_{l}}$ for some $\lambda=\left(\lambda_{1}, \ldots, \lambda_{l}\right) \in((1 / 2) Z)^{l}$ and $\operatorname{det} \rho^{*}(g)^{2}=$ $\chi_{1}^{* 2 \lambda_{1}^{*}} \ldots \chi_{l}^{* 2 \lambda_{l}^{*}}$ for some $\lambda^{*}=\left(\lambda_{1}^{*}, \ldots, \lambda_{l}^{*}\right) \in((1 / 2) \mathbf{Z})^{l}$. Since $\operatorname{det} \rho^{*}(g)=\operatorname{det} \rho(g)^{-1}$, we have $\lambda^{*}=-\lambda U$.

EXAMPLE 2.1. For simplicity, we deal with the case $n=2$ in Example 1.10. Then we have

and

$$
\begin{aligned}
& G=\left\{g=\left(\begin{array}{ll}
a & 0 \\
c & b
\end{array}\right) ; a b \neq 0\right\} \\
& V=\left\{X=\left(\begin{array}{ll}
x & y \\
y & z
\end{array}\right)\right\}
\end{aligned}
$$

The basic $K$-relative invariants of $(G, \rho, V)$ (respectively $\left(G, \rho^{*}, V^{*}\right)$ ) are $f_{1}(X)=$ $x$ and $f_{2}(X)=\operatorname{det} X$ (respectively $f_{1}^{*}(X)=z, f_{2}^{*}(X)=\operatorname{det} X$ ) corresponding to $\chi_{1}(g)=a^{2}, \chi_{2}(g)=a^{2} b^{2}$ (respectively $\chi_{1}^{*}(g)=b^{-2}, \chi_{2}^{*}(g)=a^{-2} b^{-2}$ ) for

$$
g=\left(\begin{array}{ll}
a & 0 \\
c & b
\end{array}\right)
$$

in $G$. Hence $\chi_{1}=\chi_{1}^{*} \chi_{2}^{*-1}$ and $\chi_{2}=\chi_{2}^{*-1}$ so that we have

$$
U=\left(\begin{array}{ll}
1 & -1 \\
0 & -1
\end{array}\right)
$$

Since

$$
\operatorname{det} \rho\left(\left(\begin{array}{ll}
a & 0 \\
c & b
\end{array}\right)\right)=a^{3} b^{3}
$$

we have $\lambda=\lambda^{*}=(0,3 / 2)$.

Let $\left\{\varepsilon_{1}, \ldots, \varepsilon_{\nu}\right\}$ be the complete set of representatives of $K^{\times} / K^{\times 2}$ in $K^{\times}$. Then we have $Y_{K}=Y_{1} \cup \cdots \cup Y_{\nu}$ with $Y_{i}=\left\{y \in Y_{K} ; f_{2}(y) \equiv \varepsilon_{i} \bmod K^{\times 2}\right\}(i=1, \ldots, \nu)$.

Let $\omega^{(i)}: K^{\times} \rightarrow \mathbf{C}^{\times}(i=1, \ldots, l)$ be a quasicharacter, that is, a continuous homomorphism. 
For $\omega=\left(\omega^{(1)}, \ldots, \omega^{(l)}\right)$ and the basic $K$-relative invariants $f(x)=\left(f_{1}(x), \ldots\right.$, $\left.f_{l}(x)\right)$, we write $\omega(f(x))$ instead of $\prod_{i=1}^{l} \omega^{(i)}\left(f_{i}(x)\right)$ for simplicity of notation.

Let || be the absolute value of $K$ normalised by $|\pi|=q^{-1}$ for a prime element $\pi$ where $q$ is the module of $K$. For $s=\left(s_{1}, \ldots, s_{l}\right)$, we write $\omega_{s}=\left(||^{s_{1}}, \ldots,||^{s_{l}}\right)$ so that $\omega_{s}(f(x))=\prod_{i=1}^{l}\left|f_{i}(x)\right|^{s_{i}}$.

Let $d x$ be the Haar measure on $V_{K}=K^{n}$ normalised by $\int_{R^{n}} d x=1$ where $R$ is the maximal compact subring of $K$. Since $d(\rho(g) x)=|\operatorname{det} \rho(g)| d x$ and $\omega_{\lambda}(f(\rho(g) x))=$ $|\operatorname{det} \rho(g)| \omega_{\lambda}(f(x))$, the measure $d_{Y}(x)=(d x) /\left(\omega_{\lambda}(f(x))\right)$ is a $G$-invariant measure on $Y$.

For $\Phi \in \mathfrak{S}\left(V_{K}\right)$ where $\mathfrak{S}\left(V_{K}\right)$ denotes the Schwartz-Bruhat space of $V_{K}$, we define the integral

$$
Z_{i}(\omega, \Phi)=\int_{Y_{i}} \omega(f(x)) \Phi(x) d_{Y}(x)(i=1, \ldots, \nu) .
$$

Now any quasi-character $\omega^{(i)}: K^{\times} \rightarrow \mathbf{C}^{\times}=\{z \in \mathbf{C} ; z \neq 0\}$ can be written uniquely as $\omega^{(i)}=||^{s_{i}} \cdot \phi_{i}$ for some $s_{i} \in \mathbf{C}$ and $\phi_{i}: R^{\times} \rightarrow \mathbf{C}_{1}^{\times}=\{z \in \mathbf{C} ;|z|=1\}$ where $R^{\times}$is the set of units of $R$. Put $\operatorname{Re} \omega^{(i)}=\operatorname{Re} s_{i}(i=1, \ldots, l)$. The following lemma is easy to prove and we omit the proof (see Sato [15]).

Lemma 2.2. If Re $\omega^{(i)}>\lambda_{i}(i=1, \ldots, l)$, the integral $Z_{i}(\omega, \Phi)$ is absolutely convergent and holomorphic with respect to $s=\left(s_{1}, \ldots, s_{l}\right) \in(\mathbf{C} /((2 \pi i) /(\log q)) \mathbf{Z})^{l} \cong$ $\mathbf{C}^{\times l}$ for $\omega=\left(||^{s_{1}} \cdot \phi_{1}, \ldots,||^{s_{l}} \cdot \phi_{l}\right)$.

Let $\mathfrak{S}^{\prime}\left(V_{K}\right)=\left\{z: \mathfrak{S}\left(V_{K}\right) \rightarrow \mathbf{C}\right.$, where $\mathbf{C}$ is a linear mapping $\}$ be the space of distributions on $V_{K}$. By Lemma 2.2, the mapping $\Phi \mapsto Z_{i}(\omega, \Phi)$ defines a distribution on $V_{K}$ when $\operatorname{Re} \omega^{(i)}>\lambda_{i}(i=1, \ldots, l)$.

For $\left(G, \rho^{*}, V^{*}\right)$, we can define a similar distribution $Z_{j}^{*}(\omega)(j=1, \ldots, \nu)$ given by

$$
Z_{j}^{*}(\omega)\left(\Phi^{*}\right)=Z_{j}^{*}\left(\omega, \Phi^{*}\right)=\int_{Y_{j}^{*}} \omega\left(f^{*}(y)\right) \Phi^{*}(y) d_{Y^{*}}(y) .
$$

Now we fix a non-trivial additive character $\psi: K \rightarrow \mathbf{C}_{1}^{\times}$and define the Fourier transformation $\mathfrak{S}\left(V_{K}^{*}\right) \ni \Phi^{*} \mapsto \widehat{\Phi}^{*} \in \mathfrak{S}\left(V_{K}\right)$ by $\widehat{\Phi}^{*}(x)=\int_{V_{K}^{*}} \Phi^{*}(y) \psi(\langle x, y\rangle) d y$ where $d y$ is the Haar measure on $V_{K}^{*}$ dual to a fixed Haar measure on $V_{K}$.

$$
\text { For } \omega=\left(\omega^{(1)}, \ldots, \omega^{(l)}\right) \text {, put } \omega^{*}=\omega^{U}=\left(\prod_{i=1}^{l} \omega^{(i) u_{i 1}}, \ldots, \prod_{i=1}^{l} \omega^{(i) u_{i l}}\right) \text {. }
$$

Our purpose is to show that $Z_{i}(\omega)$ and $Z_{j}^{*}(\omega)$ are continued analytically to all $\omega$ and satisfy the functional equation:

$$
\widehat{Z}_{i}(\omega)=\sum_{j=1}^{\nu} \Gamma_{i j}(\omega) Z_{j}^{*}\left(\omega^{*} \omega_{\lambda^{*}}\right)(i=1, \ldots, \nu)
$$


under some additional conditions where $\widehat{Z}_{i}(\omega)\left(\Phi^{*}\right)=Z_{i}\left(\omega, \widehat{\Phi}^{*}\right)$. Recall that $\omega_{\lambda^{*}}=$ $\left(||_{\lambda_{1}^{*}}, \ldots,||_{\lambda_{l}^{*}}\right)$ with $\operatorname{det} \rho^{*}(g)^{2}=\chi_{1}^{2 \lambda_{1}^{*}} \cdots \chi_{l}^{2 \lambda_{l}^{*}}$.

Actually when $K$ is a local field with $\operatorname{ch}(K)=0$, then (2.1) is obtained under some conditions and it is called "the fundamental theorem of P.V. over $K$ ".

\section{Rationality FOR almost all $p$}

For a rational prime $p$, let $K_{p}$ denote the local field with the constant field $\mathbf{F}_{p}$. For $f \in \mathbf{Z}\left[x_{1}, \ldots, x_{n}\right]$, we denote $(f \bmod p) \in \mathbf{F}_{p}\left[x_{1}, \ldots, x_{n}\right]$ by $f_{p}$. Then we have the following theorem which was suggested by Professor M. Kashiwara.

Theorem 3.1. For almost all p, the integral

$$
Z_{p}\left(s, \Phi_{p}\right)=\int_{K_{p}^{n}}\left|f_{p}(x)\right|_{K_{p}}^{s} \Phi_{p}(x) d_{p} x
$$

is a rational function of $t=p^{-s}$ where $\Phi_{p} \in \mathfrak{S}\left(K_{p}^{n}\right)$ and $d_{p} x$ is a Haar measure on $K_{p}^{n}$.

Proof: Let $K=\mathbf{Q}((t))$ be the field of formal power series over $\mathbf{Q}, X=\Omega^{n}$ the affine space and $X_{K}=K^{n}$. Let $f$ denote the morphism $X \rightarrow \Omega$ defined by $f \in \mathbb{Z}\left[x_{1}, \ldots x_{n}\right]$; then there exists a nonsingular algebraic variety $Y$ and a projective morphism $h: Y \rightarrow X$ both defined over $K$ with the following property: let $b$ denote an arbitrary point of $Y_{K}, \mathfrak{D}_{K}$ the local ring of $Y$ at $b$ relative to $K$ (consisting of "functions" defined over $K$ ), and $\mathfrak{M}_{K}$ the ideal of non-units of $\mathfrak{D}_{K}$; then there exists an ideal basis $\left(y_{1}, \ldots, y_{n}\right)$ of $\mathfrak{M}_{K}$, elements $u, v$ of $\mathfrak{O}_{K}-\mathfrak{M}_{K}$, and integers $N_{i} \geqq 0, \nu_{i} \geqq 1$ for $1 \leqq i \leqq n$ such that

$$
f \circ h=u \cdot \prod_{i=1}^{n} y_{i}^{N_{i}}, \quad h^{*}(d x)=v \cdot \prod_{i=1}^{n} y_{i}^{\nu_{i}-1} d y .
$$

The existence of such a pair $(Y, h)$ is guaranteed by Hironaka's theorem $[7$, p.109326]. Then for almost all $p$, the reduction modulo $p$ is well-defined and we have similar results for $K_{p}, f_{p}, \ldots$ et cetera. Then by arguments similar to those in the Appendix of Igusa [13], we obtain our result.

REMARK 3.2. Let $K$ be a number field. For $f \in \mathcal{D}_{K}\left[x_{1}, \ldots, x_{n}\right]$, we have a similar result to Theorem 3.1 for almost all prime ideals $\mathfrak{P}$ of $\mathfrak{O}_{K}$.

\section{Functional EQUATIONS}

LEMma 4.1. Let $G$ denote a locally compact totally disconnected group, $H$ a closed subgroup of $G, X=H \backslash G$, and $\omega: G \rightarrow \mathbf{C}^{\times}$a quasicharacter. Put $\xi_{X}(\omega)=$ 
$\left\{T \in \mathfrak{S}(X)^{\prime} ; g T=\omega(g)^{-1} T\right.$ for all $\left.g \in G\right\}$. Then we have $\operatorname{dim}_{\mathbf{C}} \xi_{X}(\omega) \leqq 1$. Moreover $\operatorname{dim}_{\mathbf{C}} \xi_{X}(\omega)=1$ if and only if $\left.\Delta_{G} \cdot \omega\right|_{H}=\Delta_{H}$ where $\Delta_{G}, \Delta_{H}$ denotes the module of $G, H$ respectively.

ProOF: See Igusa [11, p.1015].

Let $(G, \rho, V)$ and its dual $\left(G, \rho^{*}, V^{*}\right)$ be $K$-regular P.V.'s with \# $\rho(G)_{K} \backslash Y_{K}=$ $\nu<+\infty$ where $K$ is a local field of characteristic $p$. Then, by Proposition 1.3, we have $Y_{K}=Y_{1} \cup \cdots \cup Y_{\nu}$ and $Y_{K}^{*}=Y_{1}^{*} \cup \cdots \cup Y_{\nu}^{*}$, that is, $\# \rho^{*}(G)_{K} \backslash Y_{K}^{*}=\nu$.

As in Section 2, we can define the zeta distribution $Z_{i}(\omega, \Phi)$ (respectively $Z_{i}^{*}\left(\omega, \Phi^{*}\right)$ ) which is convergent when $\operatorname{Re} \omega^{(j)}>\lambda_{j}$ (respectively $\left.\operatorname{Re} \omega^{(j)}>\lambda_{j}^{*}\right)(1 \leqslant i \leqslant \nu$, $1 \leqslant j \leqslant l)$.

We denote by $Z_{i}(\omega)$ the distribution defined by $\Phi \mapsto Z_{i}(\omega, \Phi)$, et cetera.

Proposition 4.2. We have

(1) $Z_{j}^{*}\left(\omega^{*} \omega_{\lambda^{*}}\right) \in \xi_{Y_{j}^{*}}\left(\omega^{*} \omega_{\lambda^{*}}\right)$ and

(2) $\widehat{Z_{i}}(\omega) \in \xi_{Y_{j}^{*}}\left(\omega^{*} \omega_{\lambda^{*}}\right)$

$(i, j=1, \ldots, \nu)$.

Proof: By a direct calculation, we obtain our results.

Proposition 4.3. Let $K$ be a local field of characteristic $p>0$ with the module $q$. For $\omega=\left(\omega^{(1)}, \ldots, \omega^{(l)}\right)$ with $\omega^{(i)}=\omega_{s_{i}} \cdot \phi_{i}\left(\phi_{i}(\pi)=1\right.$ for a prime element $\pi)$, assume that $Z_{i}(\omega, \Phi)$ and $Z_{j}^{*}\left(\omega, \Phi^{*}\right)$ are rational functions of $q^{-s_{1}}, \ldots, q^{-s_{l}}$. Then for all $\Phi^{*} \in \mathfrak{S}\left(Y_{K}^{*}\right)$, we have

$$
Z_{i}\left(\omega, \widehat{\Phi}^{*}\right)=\sum_{j} \Gamma_{i j}(\omega) Z_{j}^{*}\left(\omega^{*} \omega_{\lambda^{*}}, \Phi^{*}\right)
$$

for $i, j=1, \ldots, \nu$.

Proof: Since $Z_{i}(\omega, \Phi)$ and $Z_{j}\left(\omega, \Phi^{*}\right)$ are rational functions, it is defined for all $\omega$ except poles and hence by Lemma 4.1 and Proposition 4.2, we have our result.

THEOREM 4.4. Let $(G, \rho, V)$ be a $K$-regular P.V. satisfying the following conditions:

(C1) its dual $\left(G, \rho^{*}, V^{*}\right)$ is a $K$-regular P.V. such that \# $\rho^{*}(G)_{K} \backslash V_{K}^{*}<+\infty$,

(C2) for $x \in S_{K}^{*}$, there exists $\chi \in X_{1}(G)_{K}$ satisfying $\chi\left(G_{x, K}\right) \nsubseteq R^{\times}$where $R^{\times}$is the set of units of the maximal compact subring $R$ of $K$, and

(C3) $Z_{j}(\omega, \Phi)$ is a rational function of $q^{-s_{1}}, \ldots, q^{-s_{l}}$ where $\omega=\left(\omega^{(1)}, \ldots, \omega^{(l)}\right)$ with $\omega^{(i)}=\omega_{s_{i}}(1 \leqq i \leqq l)$.

Then we have the functional equation

$$
Z_{i}\left(\omega, \widehat{\Phi}^{*}\right)=\sum_{j} \Gamma_{i j}(\omega) Z_{j}^{*}\left(\omega^{*} \omega_{\lambda^{*}}, \Phi^{*}\right)
$$


for all $\Phi^{*} \in \mathfrak{S}\left(V_{K}^{*}\right)$ for $i, j=1, \ldots, \nu$ where $\nu=\# \rho^{*}(G)_{K} \backslash Y_{K}^{*}$.

Proof: The condition (C2) corresponds to Sato [15, Lemma 2.2, p.474] for the case of $\operatorname{ch}(K)=0$. Then the proof is similar to the case of $\operatorname{ch}(K)=0$ (using Proposition 4.3). (See Igusa [11] and Sato $[15$, p.477]).

Now let $(G, \rho, V)$ be a reductive Q-regular P.V. Then for almost all $p$, we have the reduction modulo $p$ and we obtain the $K_{p}$-regular P.V. $\left(G_{p}, \rho_{p}, V_{p}\right)$ where $K_{p}$ is a local field with the constant field $\mathbf{F}_{p}$.

Assumption A. Assume that \# $\rho_{p}(G)_{K_{p}} \backslash S_{K_{p}}<+\infty$ and for $x \in S_{K_{p}}$, there exists $\chi \in X_{1}\left(G_{p}\right)_{K_{p}}$ satisfying $\chi\left(G_{p, x, K_{p}}\right) \nsubseteq R_{p}^{\times}$for almost all $p$.

Let $(G, \rho, V)$ be a reductive Q-regular P.V. with Assumption A. Let $f_{1}, \ldots, f_{l}$ be basic $Q$-relative invariants with $\mathbf{Z}$-coefficients. Denote $\left|f_{1} \bmod p\right|_{K_{p}}^{s_{1}} \cdots\left|f_{l} \bmod p\right|_{K_{p}}^{s_{l}}$ by $\left|f^{(p)}(x)\right|_{K_{p}}^{s}$ and put $Z_{i}^{p}\left(s, \Phi_{p}\right)=\int_{\left(Y_{K_{p}}\right)_{i}}\left|f^{(p)}(x)\right|_{K_{p}}^{s} \Phi_{p}(x) d_{Y_{p}}(x)$ for $\Phi_{p} \in \mathfrak{S}\left(V_{K_{p}}\right)$.

TheOREM 4.5. Let $(G, \rho, V)$ be a reductive Q-regular P.V. with Assumption $A$. Then for almost all rational primes $p$, the integral $Z_{i}^{p}\left(s, \Phi_{p}\right)\left(i=1, \ldots, \nu_{p}, Y_{K_{p}}\right.$ $\left.=Y_{1} \cup \cdots \cup Y_{\nu_{p}}\right)$ is a rational function and satisfies the functional equation:

$$
Z_{i}^{p}\left(s, \widehat{\Phi_{p}}\right)=\sum_{j=1}^{\nu_{p}} \Gamma_{i j}(s) Z_{j}^{p}\left(s^{*}, \Phi_{p}\right)
$$

$\left(i=1, \ldots, \nu_{p}\right)$.

When $l=1$, we have $s^{*}=(n / d)-s$ with $n=\operatorname{dim} V$ and $d=\operatorname{deg} f$. In general, for $\omega=\omega_{s}=\omega_{s_{1}} \cdots \omega_{s_{l}}$, we have $\omega_{s^{*}}=\omega^{*} \omega_{\lambda^{*}}$.

ProOF: By Theorem 4.4 and using the results of Section 1 and Section 3, we obtain our result.

\section{REFERENCES}

[1] A. Borel, Collected papers.

[2] A. Borel, Linear algebraic groups (2nd edition), Graduate texts in mathematics (SpringerVerlag, Berlin, Heidelberg, New York, 1991).

[3] F. Bruhat and J. Tits, 'Groupes algebriques sur un corps local, Chapitre III, complements et applications a la cohomologie Galoisienne', J. Fac. Sci. Univ. Tokyo Sect. 1A Math. 34 (1987).

[4] Z. Chen, 'Fonction zêta associée à un espace préhomogène et sommes de Gauss'.

[5] Z. Chen, 'A classification of irreducible prehomogeneous vector spaces over an algebraically closed field of characteristic p (II)', (in Chinese), Chinese Ann. Math. Ser. A. 9 (1988), p. 10-22.

[6] A. Gyoja, 'Talk at 1993 JAMI conference', Johns Hopkins University. 
[7] H. Hironaka, 'Resolution of singularities of an algebraic variety over a field of characteristic zero', Ann. of Math 79 (1964), p. 109-326.

[8] J.-I. Igusa, 'Complex powers and asymptotic expansion I', J. Reine Angew. Math 268/269 (1974), 110-130.

[9] J.-I. Igusa, 'Complex powers and asymptotic expansion II', J. Reine Angew.Math 278/279 (1975), 308-321.

[10] J.-I. Igusa, Lectures on forms of higher degree (Tata Inst. Fund. Research, Bombay, 1978).

[11] J.-I. Igusa, 'Some results on p-adic complex powers', Amer. J. Math 106 (1984), 1013-1032.

[12] J.-I. Igusa, 'Zeta distributions associated with some invariants', Amer. J. Math 110 (1988), 197-233.

[13] J.-I. Igusa, 'Some observations on higher degree characters', Amer. J. Math 99 (1977), $393-417$.

[14] F. Sato, 'Zeta functions in several variables associated with prehomogeneous vector spaces I: Functional equations', Tôhoku Math. J. 34 (1982), 437-483.

[15] F. Sato, 'On functional equations of zeta distributions', Adv. Stud. Pure Math. 15 (1989), 465-508.

[16] M. Sato and T. Kimura, 'A classification of irreducible prehomogeneous vector spaces and their relative invariants', Nagoya Math. J. 65 (1977), 1-155.

[17] M. Sato and T. Shintani, 'On zeta functions associated with prehomogeneous vector spaces', Ann. of Math 100 (1974), 131-170.

[18] J.P. Serre, Cohomologie Galoisienne, Lecture Notes in Math. 5 (Springer-Verlag, Berlin, Heidelberg, New York, 1964). 